\title{
FOURIER TRANSFORMS WHICH ARE ASYMPTOTICALLY CAUCHY DENSITIES*
}

\author{
BY
}

\section{L. CAMPBELL}

Queen's University

During an investigation [2] of the phase distribution of filtered Gaussian noise, two integrals of the form

$$
f(x, b)=\int_{-\infty}^{\infty} g(y, b) e^{-b|y|+i x y} d y
$$

were encountered, where $b$ is a large positive number. If the exponential term dominates the behavior of the integrand it is possible to get a simple approximation for $f$ as $b \rightarrow \infty$. TheOREM. Let $g(\cdot, b)$ be bounded and measurable on $\mathbb{R}$ and continuous at zero for all $b>B$. Let $g$ satisfy the condition

$$
|g(y, b)-g(0, b)| \leqslant K|y|^{p}
$$

for $y \in(-\delta, \delta)$ and all $b>B$. Let $B \geqslant 0, \delta>0$, and $p>0$. Let $|g(y, b)| \leqslant M$ for $y \in \mathbb{R}$ and $b>B$ and let $B_{1}=\max (B, p / \delta)$. If $f$ is defined by (1), then

$$
\left|f(x, b)-\frac{2 b g(0, b)}{x^{2}+b^{2}}\right| \leqslant\left[2 K \Gamma(p+1)+4 M B_{1}^{p} e^{-B_{1} \delta}\right] b^{-p-1} .
$$

Proof. From (1),

$$
f(x, b)-\frac{2 b g(0, b)}{x^{2}+b^{2}}=J_{1}+J_{2}
$$

where

$$
J_{1}=\int_{-\delta}^{\delta} e^{-b|y|+i x y}[g(y, b)-g(0, b)] d y
$$

and

$$
J_{2}=\int_{|y|>\delta} e^{-b|y|+i x y}[g(y, b)-g(0, b)] d y .
$$

${ }^{*}$ Received September 10, 1985. 
It follows from (2) and elementary manipulations that

$$
\left|J_{1}\right| \leqslant 2 K \int_{0}^{\infty} e^{-b y} y^{p} d y=2 K \Gamma(p+1) b^{-p-1} .
$$

Similarly,

$$
\left|J_{2}\right| \leqslant 4 M \int_{\delta}^{\infty} e^{-b y} d y=4 M b^{-1} e^{-b \delta}
$$

Thus

$$
b^{p+1}\left|J_{1}+J_{2}\right| \leqslant 2 K \Gamma(p+1)+4 M b^{p} e^{-b \delta} .
$$

Since $b^{p} e^{-b \delta}$ takes its maximum value at $b=p / \delta$, the result follows.

The function $b / \pi\left(x^{2}+b^{2}\right)$ is, as a function of $x$, the Cauchy probability density function with interquartile range $2 b$.

As one illustration, consider

$$
F(x, a)=\int_{-\infty}^{\infty} I_{|y|}(a) e^{i x y} d y
$$

where $0<a<2$ and $I_{|y|}(a)$ denotes a modified Bessel function of order $|y|$ and argument a. Cooke [1] has expressed the integral in (3) in terms of an integral of two Cauchy densities. Here, we obtain an approximate value of $F(x, a)$ for small $a$. If we put $b=\ln (2 / a)$ we have $b>0$ and

$$
I_{|y|}(a)=e^{-b|y|} g(y, b),
$$

where

$$
g(y, b)=\frac{1}{\Gamma(|y|+1)} \sum_{n=0}^{\infty} \frac{e^{-2 n b}}{n !(1+|y|) \cdots(n+|y|)} .
$$

Thus the integral in (3) is of the same form as (1). Moreover, the series above is bounded for all $|y|$ and all $b \geqslant 0$ by its value at $b=y=0$, which is $I_{0}(2)$. Since $1 / \Gamma(|y|+1)$ is bounded above by 1.12867 , we have $|g(y, b)| \leqslant M$, where $M=2.573$.

To obtain a condition of the form (2), we begin with

$$
g(0, b)-g(y, b)=\sum_{n=0}^{\infty} \frac{e^{-2 n b}}{n !}\left[\frac{1}{\Gamma(n+1)}-\frac{1}{\Gamma(n+1+|y|)}\right] .
$$

Because of the convexity of the gamma function, it is elementary to show that, for $|y| \leqslant 1$,

$$
\Gamma(n+1+|y|)-\Gamma(n+1) \leqslant n \Gamma(n+1)|y|,
$$

and thus that

$$
\frac{1}{\Gamma(n+1)}-\frac{1}{\Gamma(n+1+|y|)} \leqslant \frac{n|y|}{\Gamma(n+1)} .
$$

From (4), and a little algebraic manipulation,

$$
g(0, b)-g(y, b) \leqslant K|y|,
$$

where $K=I_{1}(2)$, the modified Bessel function of order one and argument two. 
Thus, by the theorem with $p=1, \delta=1, B=0$,

$$
\left|F(x, a)-\frac{2 b I_{0}\left(2 e^{-b}\right)}{x^{2}+b^{2}}\right| \leqslant \frac{6.97}{b^{2}},
$$

and, since $I_{0}(0)=1$,

$$
F(x, a) \doteqdot \frac{2 \ln (2 / a)}{x^{2}+[\ln (2 / a)]^{2}}
$$

for values of $a$ close to zero.

Acknowledgment. This result grew out of joint work with G. Swanson and P. H. Wittke. The research was supported by the Natural Sciences and Engineering Research Council of Canada, grant No. A2151.

\section{REFERENCES}

[1] J. C. Cooke, Note on some integrals of Bessel functions with respect to their order, Monatsh. Math. 58, 1-4 (1954)

[2] G. D. Swanson, A study of the stochastic phase in digital frequency modulation, M. Sc. thesis, Department of Electrical Engineering, Queen's University (August 1985) 